\title{
Association of index finger palpatory assessment of pubovisceral muscle body integrity with MRI-documented tear
}

\author{
Ying Sheng PhD, MSN, RN ${ }^{1}$ ～Lisa Kane Low PhD, CNM, FACNM, FAAN ${ }^{1,2,3}$ \\ Xuefeng Liu PhD ${ }^{1}$ | James A. Ashton-Miller PhD ${ }^{4}$ Janis M. Miller PhD, APRN, FAAN ${ }^{1,3}$
}

${ }^{1}$ University of Michigan School of Nursing, Ann Arbor, Michigan

${ }^{2}$ Department of Women's Studies, University of Michigan, Ann Arbor, Michigan

${ }^{3}$ Department of Obstetrics and Gynecology, School of Medicine, University of Michigan, Ann Arbor, Michigan

${ }^{4}$ Department of Mechanical Engineering, University of Michigan College of Engineering, Ann Arbor, Michigan

\section{Correspondence}

Janis M Miller, School of Nursing, University of Michigan, $400 \mathrm{~N}$ Ingalls, Ann Arbor, MI 48109.

Email: janismm@umich.edu

\section{Funding information}

Research on Women's Health Specialized Center of Research (SCOR) on Sex and Gender Factors Affecting Women's Health and National Institute on Child and Human Development; National Institute on Child and Human Development

\begin{abstract}
Aims: Pubovisceral (PV) muscle tears are associated with pelvic floor disorders. The goal of this study was to determine whether index finger palpatory assessment of PV muscle body integrity through the lateral vaginal wall is a reliable indicator of PV muscle tear severity diagnosed by magnetic resonance imaging (MRI).
\end{abstract}

Methods: We studied 85 women, 7 weeks after vaginal birth. All had at least one risk factor for obstetric-related PV muscle tear. The ordinal outcome measure of MRI-documented PV muscle tear was defined as: none, less than $50 \%$ unilateral tear, $50 \%$ or greater unilateral tear or less than $50 \%$ bilateral tear, and $50 \%$ or greater bilateral tear. PV muscle body integrity by palpatory assessment was scored on a matrix, with each side scored independently and classified as PV muscle body "present" (assuredly felt), "equivocal” (not sure if felt), or "absent" (assuredly not felt). Proportional odds models were constructed to estimate the relationship between PV muscle body integrity palpatory assessment and MRI-documented PV muscle tears.

Results: Thirty-five percent of study participants exhibited varying degrees of MRI-documented PV muscle tears. Using palpatory assessment, we identified "PV muscle body present bilaterally" in $20 \%$, "equivocal unilaterally or present contralaterally" in $8 \%$, "equivocal or absent unilaterally" or "equivocal bilaterally" in $62 \%$, and "absent bilaterally" in $9 \%$. The odds ratio for estimating MRI results from palpatory assessment was $3.62(95 \%$ confidence interval $=$ 1.70-7.73, $P=0.001$ ).

Conclusions: A rapid and inexpensive palpatory assessment in the clinic was highly associated with the risk of MRI-documented PV muscle tear and is a useful component of a clinical assessment.

\section{K E Y W O R D S}

birth injuries, levator ani tear, muscle evaluation, palpation, pelvic floor muscles, physical assessment, postpartum women 


\section{INTRODUCTION}

The pubovisceral (PV) muscle, also known as pubococcygeus, is the portion of the levator ani muscle most vulnerable to sustaining a tear from the pubic bone during vaginal birth. ${ }^{1-3}$ The PV muscle is at high risk for stretch-related tear, ${ }^{4}$ likely due to a multitude of interacting factors. Variations in maternal pelvic shape, fetal head shape, the degree of molding during delivery, symphyseal diastasis, and presentation may all affect the maximum muscle stretch ratios, increasing the risk of muscle tear. ${ }^{5}$ The prevalence of PV muscle tear ranges from $13 \%$ to $36 \%$ of women who have had a vaginal delivery. ${ }^{6-8} \mathrm{~A}$ higher prevalence of PV muscle tear has been associated with older maternal age and obstetric variables indicative of a more complex vaginal birth. ${ }^{8-12}$

For the purposes of this paper, a PV muscle tear is characterized on magnetic resonance imaging (MRI) by a discontinuity or visible loss of muscle bulk that can be observed. ${ }^{9,13}$ A complete PV muscle tear is defined as a full disruption of its origin at the pubic bone.

A PV muscle tear has been identified as a risk factor for two important pelvic floor disorders: pelvic organ prolapse and stress urinary incontinence (SUI), though the latter remains controversial. ${ }^{9,14}$ For decades, the predominant nonsurgical treatment of SUI and pelvic organ prolapse has focused on the concept of increasing the strength of the levator ani muscle complex that surrounds the genital hiatus to compensate for any prior injury. It is noteworthy that the use of strengthening exercises, known as pelvic muscle exercises, have generally been prescribed in the presence of a possible PV muscle tear; however, there is a lack of evidence as to whether women with a documented PV muscle tear actually benefit from pelvic muscle exercises in comparison with their peers whose PV muscle is not torn. This physiologic reality may logically explain why certain women report that, even though they follow pelvic muscle exercise regimens, they fail to improve on measures of pelvic muscle strength, regardless of their effort.

MRI, as well as ultrasound imaging tests, have been used to identify PV muscle tears in research studies, but rarely in clinical settings. ${ }^{7,11,15}$ This is, at least in part, due to lack of selection criteria concerning individual need for these high-cost diagnostic measures. The current state is that postpartum women, even with clear risk factors for PV muscle tear at childbirth, rarely receive any assessment at all for PV muscle tear postpartum.

Whether symptomatic or not in the postpartum period, the missing piece in the chain of evaluation (from identification of delivery-related risk factors to determination of whether a woman, in actuality, experienced a PV muscle tear) is an agreed-upon, specific, low cost, and validated clinical examination assessment. The aim of this study was to determine the extent to which assessment of the PV muscle body integrity using index finger palpatory assessment at the muscle site where it passes the vaginal sidewall bilaterally is predictive of MRI results.

\section{2 | MATERIALS AND METHODS}

\section{1 | Study design}

The results reported here were obtained from a planned sub-analysis of a parent study, Evaluating Maternal Recovery from Labor and Delivery (EMRLD). EMRLD is a longitudinal observational cohort study initiated on 14 January 2004 and ended on 1 April 2012 with data collection intervals at 7 weeks and 8 months postpartum. The main aim of EMRLD was to study the postvaginal birth recovery of the PV muscle (the anterior portion of the levator ani muscle often known colloquially as the "Kegel muscle"). Main results were previously reported. ${ }^{9,12}$ In this study, only data at 7 weeks postpartum were used because PV muscle tears essentially remained unchanged at 8 months, and data at 7 weeks have a larger sample size. Written informed consent was signed by all participants before study participation.

\subsection{Study sample}

A flowchart of study participation selection (Figure 1) portrays the parent study criteria for recruitment of 95 women enrolled in the parent study. All women were required to be over 18 years and able to read and sign the consent forms. All had to have at least one risk factor for PV muscle tear, including maternal age greater than 33 years, second stage of labor longer than 150 minutes or less than 30 minutes, delivered infant weighing greater than $4000 \mathrm{gm}$, forceps or vacuum delivery, or third- or fourth-degree anal sphincter laceration. Exclusion criteria for the parent study included prior urogynecologic surgery, unwillingness to undergo a pelvic examination, history of neurological conditions or traumatic accident injury, medical conditions (such as connective tissue disorders), or that English was not the primary health care language for the patient. Additional criteria were applied for this study. Of the 95 participants from the parent study, five were excluded due to lack of an interpretable MRI, and five due to lack of palpatory assessment data, with one due to pain. Thus, 85 postpartum women comprised the final sample. 


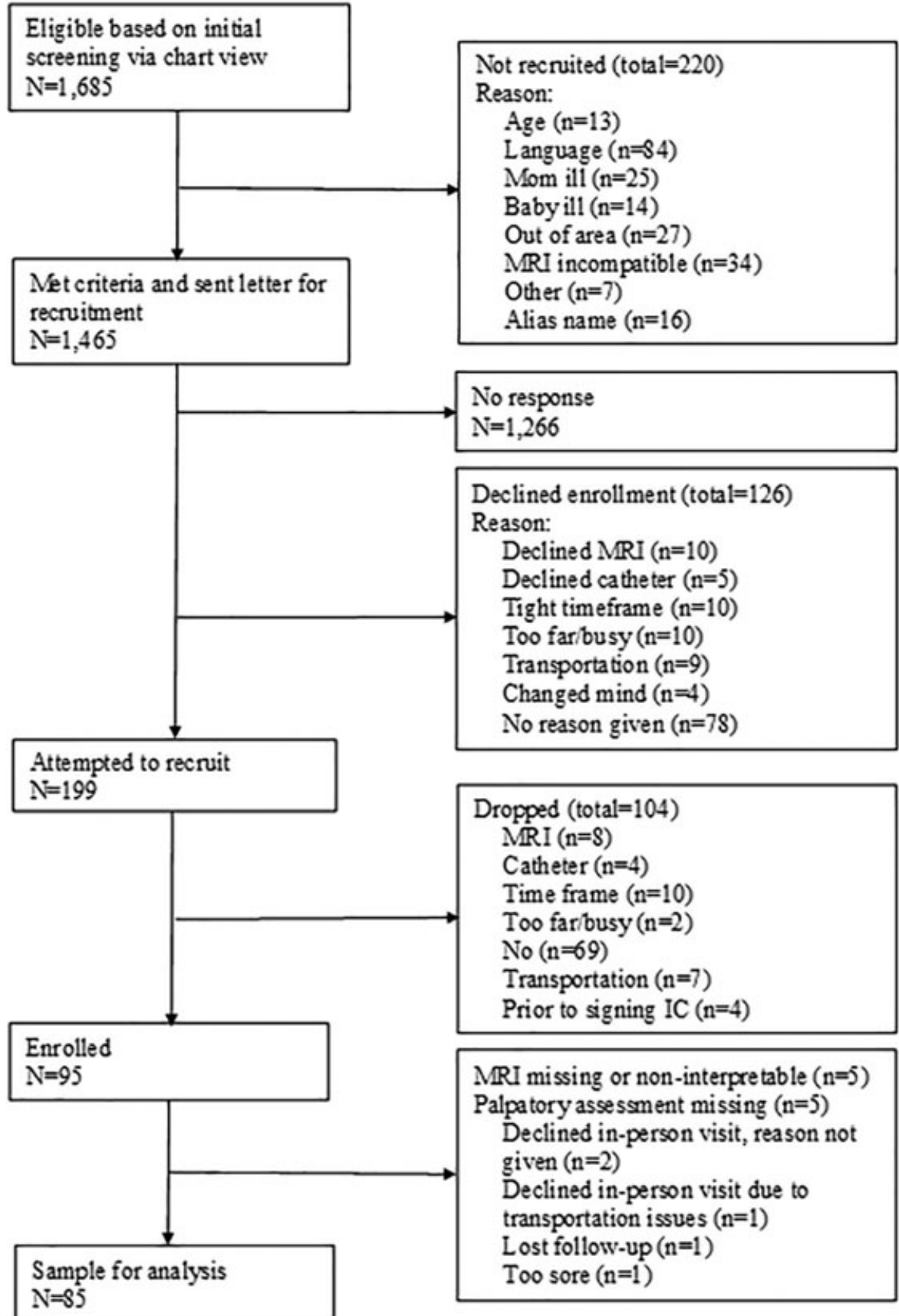

FIG URE 1 Flow diagram for study participant selection. IC, informed consent; MRI, magnetic resonance imaging

\subsection{Index finger palpatory assessment of PV muscle body integrity: predictor variable}

We use the phrase "index finger palpatory assessment of PV muscle body integrity" to distinguish from "pelvic floor muscle digital assessment," such as the Brink digital examination. Although they both are physical examination skills using palpation, the former is used to assess for muscle body integrity, while the latter ${ }^{16}$ assumes integrity and assesses for muscle strength.

For index finger palpatory assessment, the index finger was placed at the expected anatomical location of the mid-muscle body of the PV as felt about $2 \mathrm{~cm}$ inside the vagina, with the finger curled to the right or left against the vaginal sidewall (Figure 2). The finger was lightly pressed against the vaginal sidewall. Sweeping slightly up and down using the finger pad to palpate for fullness of the PV muscle body on one side and then on the other side, attempting to determine if the body of the muscle can be clearly felt with the woman at rest. If the body of the muscle cannot be clearly felt, the above procedure can be repeated while the woman attempts a pelvic muscle contraction. Ultimately, the status of the PV muscle body was determined during palpatory assessment by degree of bulk felt as continuous soft 


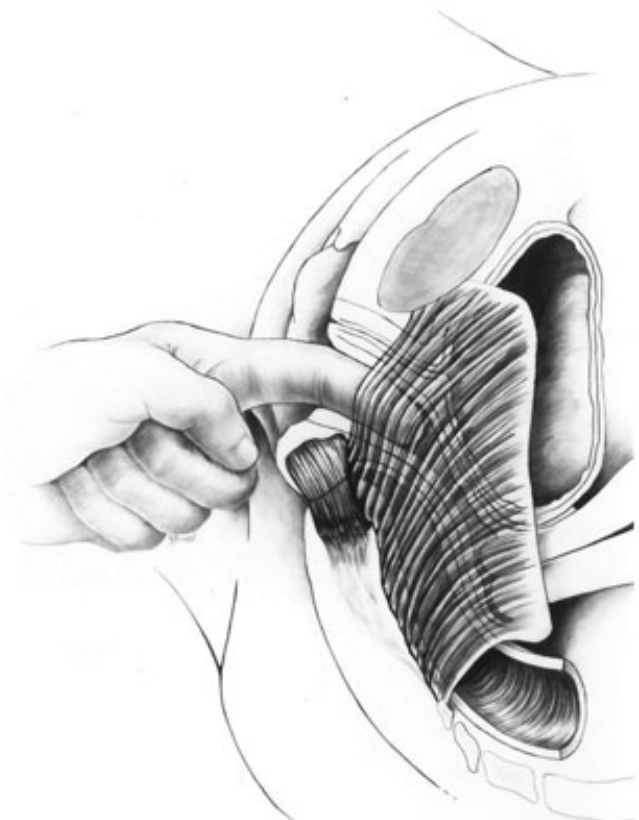

(c) Delancey 1992

FIG URE 2 Diagrammatic portrayal of index finger placement for palpatory assessment of the PV muscle body integrity, (C) DeLancey, used with permission. PV, pubovisceral

tissue resistance interposed between the lateral vaginal wall and the bony pubic rami. Absence of the muscle body will be felt as a bony area at the inferior pubic ramus, and there will be pronounced thinness of the PV muscle more dorsally.

To record findings of the palpatory assessment, the clinician chooses between three categories per palpatory assessment of each side. If the body of the muscle could definitively be felt on palpatory assessment, that side was graded as "PV muscle present". If the muscle body could not be felt, and instead there was a sense of indentation and/or feeling of hardness of the pubic ramus, the palpatory assessment for that side was graded as PV muscle "absent". If the examiner was unable to confidently ascertain that the PV muscle body was either "present" or "absent", the finding was scored as "equivocal". The process for scoring was repeated on the contralateral side.

\subsection{Dependent variable: PV muscle tear status assessed via MRI}

PV muscle tear status, measured via MRI, served as the dependent variable in this study. MRI has been the definitive test to identify PV muscle tears, and details of the MRI procedures used for the parent study of EMRLD are published elsewhere. ${ }^{9,13}$ In brief, a 3T Philips Achieva (Philips Medical System, Eindhoven, The Netherlands) with an eight-channel cardiac coil was used, and $2 \mathrm{~mm}$ images were obtained in the coronal, axial, and sagittal planes. The scoring from the MRI assessment for PV muscle tear by a musculoskeletal radiologist was categorized into five categories for each side of the muscle: no tear, subtle tear, less than $50 \%$ tear, more than $50 \%$ tear, and complete tear.

\section{5 | Procedures}

In this study, the examiner who performed the index finger palpatory assessment was a women's health nurse practitioner who had experience in performing the same assessment in approximately 200 nonpostpartum incontinent women in a previous study. The examiner did not have any unique training or exposure to additional education beyond her graduate degree. Thus, this examiner was a representative of general women's health primary care providers.

The MRIs were read by a board-certified, fellowshiptrained musculoskeletal MRI radiologist who was blinded to birth data, risk category, and palpatory assessment results for PV muscle body integrity. ${ }^{9,13}$

\section{6 | Data management and analysis}

Data from the index finger palpatory assessment scoring matrix from both sides was classified into four grades that could represent an individual's overall PV status: PV muscle body present bilaterally (coded as "0"), equivocal unilaterally and present on the contralateral side (coded as " 1 "), equivocal bilaterally or absent unilaterally (coded as "2"), and absent bilaterally (coded as " 3 ").

Data from both PV muscle sides per MRI evaluation was also categorized into four grades: no tear or subtle bilateral tear (coded as "none"), less than 50\% unilateral tear (coded as "grade I tear"), $50 \%$ or greater unilateral tear or less than $50 \%$ bilateral tear (coded as "grade II tear"), and $50 \%$ or greater bilateral tear (coded as "grade III tear")., ${ }^{9,12}$

Descriptive statistics were used to characterize the sample variables, including maternal age, education level, race, PV muscle status by palpatory assessment and by MRI, and birth-related risk factors for PV muscle tear. Means, standard deviations, and ranges were calculated for continuous variables, and frequencies and percentages were calculated for categorical variables.

A proportional odds model ${ }^{17}$ was used to estimate the association between PV muscle body integrity by index finger palpatory assessment and PV muscle tear by MRI by calculating the odds of higher-grade category of MRIdocumented PV muscle tear relative to the lower-grade category contributed by each increasing grade in the palpatory assessment measure.

All the analyses were performed using SPSS 23 (SPSS Inc, Chicago, IL). The significance level was chosen as $P<0.05$. 
TABLE 1 Characteristics of postpartum women at high risk for PV muscle tear $(\mathrm{N}=85)$

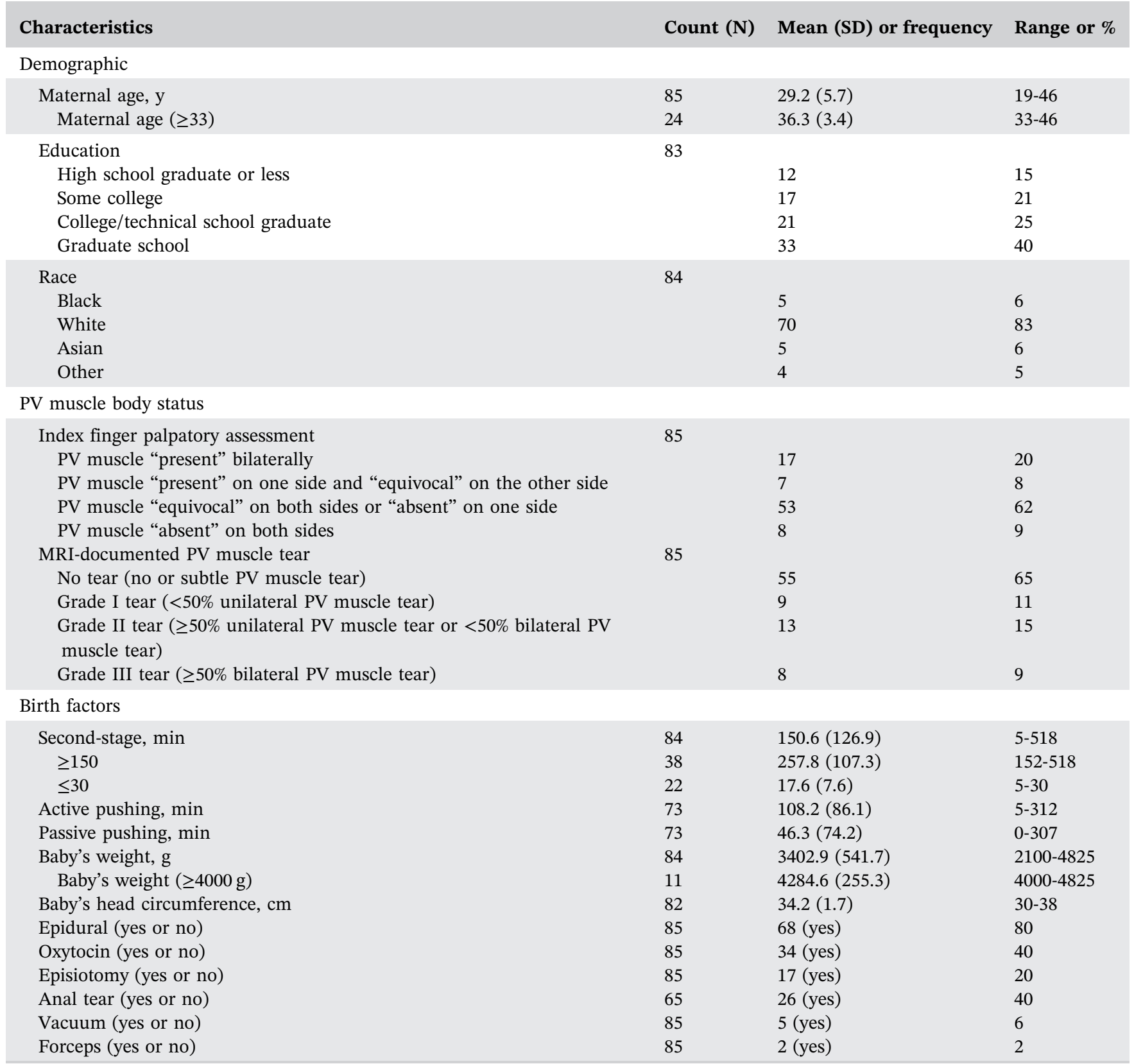

Abbreviations: MRI, magnetic resonance imaging; PV, pubovisceral.

\section{$2.7 \quad$ Ethics statement}

This study was approved by the Institutional Review Board of the University of Michigan.

\section{3 | RESULTS}

\section{1 | Final sample description}

Descriptive characteristics of the 85-women study sample, including distribution of obstetric-related risk factors for PV muscle tear, are presented in Table 1. Average age of the study sample was 29.2 years. Twenty-four women were older than 33 years, with mean age 36.3 years. The majority $(65 \%)$ of women had a college education or higher. Most women were white (83\%).

Nine percent of women were identified by palpatory assessment as PV muscle body "absent" bilaterally, while $20 \%$ of women were identified as PV muscle body "present" bilaterally. Thirty-five percent of women had MRI-documented PV muscle tear with varying degrees of severity represented. 
TABLE 2 The association of palpated PV muscle body integrity with MRI-documented PV muscle tear status among women at high risk for PV muscle tear $(\mathrm{N}=85)$

$\begin{array}{llll} & \text { OR } & \text { 95\% CI } & \text { P value } \\ \text { Index finger palpatory } & 3.62 & 1.70-7.73 & 0.001 \\ \text { assessment for estimating odds } \\ \text { of PV muscle tear }{ }^{\mathrm{a}}\end{array}$

Abbreviations: CI, confidence interval; MRI, magnetic resonance imaging; OR, odds ratio; PV, pubovisceral.

${ }^{\text {a }}$ Treated as a continuous scale (0 to 3 ), 0 indicates PV muscle body present and 3 indicates that PV muscle body is not palpable.

\section{2 | Bivariate proportional odds model results}

Results of the proportional odds model exploring the index finger palpatory assessment as an estimate of MRIdocumented PV muscle tear severity are shown in Table 2. On average, the estimated odds of having a high-grade MRI-documented PV muscle tear category relative to lower category increased by 3.62 (95\% confidence interval $=1.70-7.73, P=0.001$ ) times for each grade increase in PV muscle tear assessed by index finger palpatory assessment.

\section{4 | DISCUSSION}

The results show that PV muscle palpatory assessment, as conducted by an experienced women's health nurse practitioner feeling for the body of the PV muscle through the lateral vaginal wall, was a statistically significant estimator of PV muscle tear severity, as shown on MRI. It should be noted that these findings hold for women at high risk for PV muscle tear due to a recent complex vaginal birth. The frequency of the "any tear" results corroborate an earlier report that used ultrasound determination of PV muscle tear. ${ }^{18}$ Both ultrasound and MRI can identify complete PV muscle tear well; however, MRI may be more precise in differentiating the broader spectrum of severity grades. ${ }^{19}$

Gainey, ${ }^{20,21}$ a highly experienced obstetrician, published his use of index finger palpatory assessment to estimate presence of PV muscle tear in 1943, reporting on 1000 patients. He found that $20 \%$ of primiparous women had an absence of the muscle, which he called a tear, during palpation. Although that was 75 years ago, it is striking that Gainey's estimate of tears in a population of women who gave birth remains consistent with the percentages found today using MRI and ultrasound. ${ }^{1,2}$ That is not only a testament to Gainey's skill, but also an indicator that his techniques deserve attention today.

Although it is now rare to use palpatory assessment alone to diagnose PV muscle tear, ${ }^{22}$ Dietz and Shek $^{23}$ have documented that muscle tear is likely identifiable by palpation. In addition, van Delft et $\mathrm{al}^{15}$ used palpatory assessment to check for PV muscle tear by assessing the presence or absence of the PV muscle body. They found that, after appropriate training, the technique can be readily learned and reliably incorporated into the clinical and research settings. Since the results of palpatory assessment may be influenced by the observers' experience and knowledge, more precise and costlier tests, such as ultrasound and MRI, are needed to confirm the diagnostic results of palpatory assessment, depending on the degree of uncertainty of the provider and the purpose of diagnosis.

Some special obstetrical factors during vaginal birth may play a critical role in PV muscle tear. For example, Low et $\mathrm{al}^{12}$ have reported that birth risk factors, including older maternal age and longer second-stage active pushing, increased the likelihood of severe PV muscle tear in this same study population. A conceptual idea (Figure 3) is that women who experienced recent vaginal birth and had additional risk factors (eg, maternal age $\geq 33$ years, second-stage labor lasting $\geq 150$ minutes or $\leq 30$ minutes, infant weight $\geq 4000 \mathrm{~g}$ ) may especially benefit from a simple, quick index finger palpatory assessment to estimate any equivocal or absent $\mathrm{PV}$ muscle body.

With the proper anatomic knowledge, which is an imperative, Gainey's technique of active index finger palpatory assessment reintroduced here is easy to learn and can be an effective aid in providing feedback to patients. Clinicians can acquire proficiency in this examination through proper training and provide a timely initial estimate of the PV muscle body status post-birth. Previous studies found that palpatory assessment had acceptable reliability to identify PV muscle tear for clinical incorporation after training in the technique. ${ }^{15}$

Although several methods exist to evaluate PV muscle functional status, such as the instrumented speculum, modified Oxford scale, and Brink digital palpation, these methods are designed to measure vaginal closure force as a response to volitional pelvic muscle contraction, while palpatory assessment (at rest or during attempted volitional pelvic muscle contraction) is intended to evaluate anatomical status. Moreover, unlike MRI, palpatory assessment is low cost and is readily available, even in limited resource settings. 


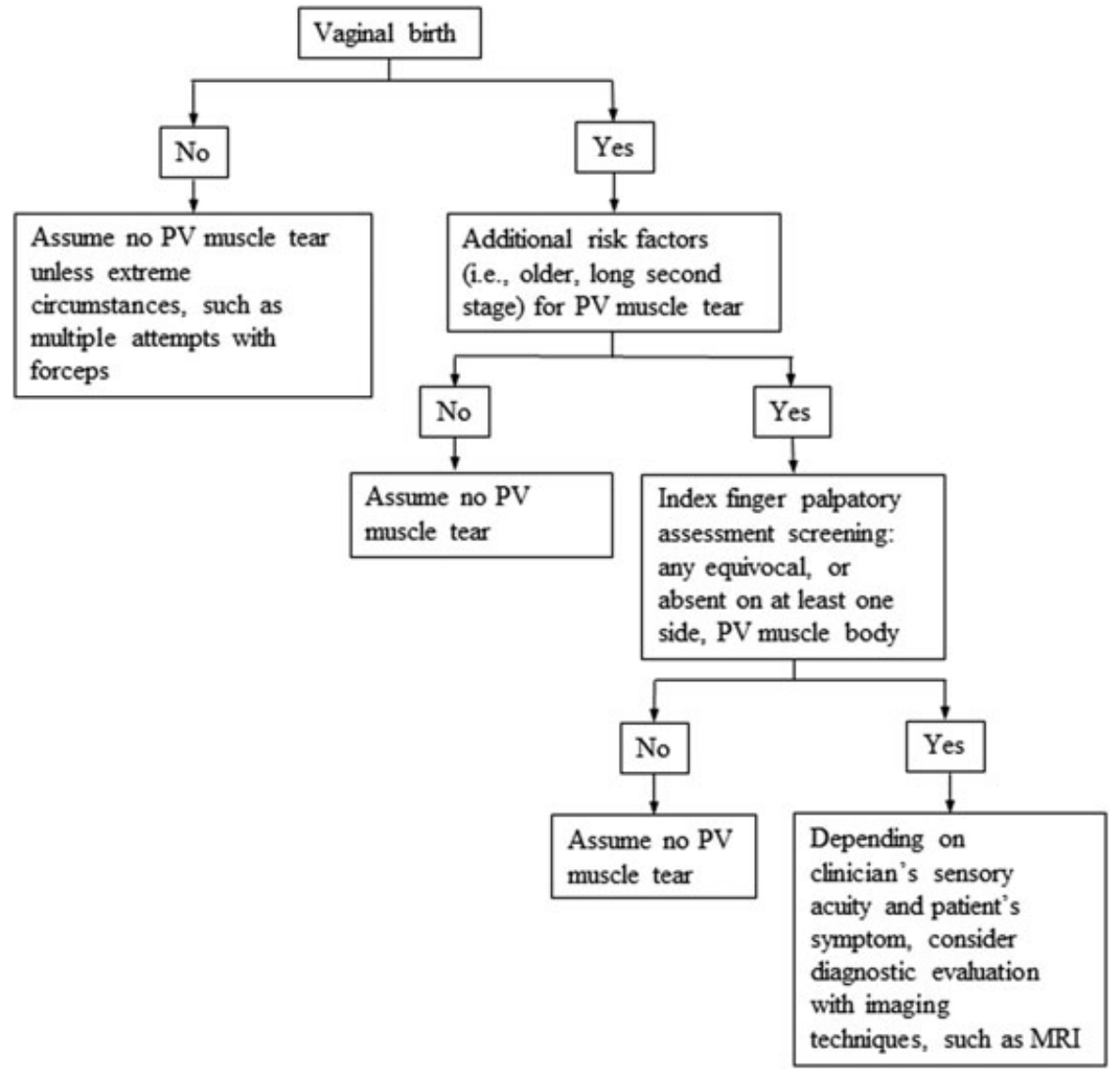

F I G U RE 3 Conceptual model of who might benefit from index finger palpatory assessment to clinically estimate PV muscle tear after childbirth. MRI, magnetic resonance imaging; PV, pubovisceral
PV muscle body integrity status rated other than "PV muscle assuredly present" by palpatory assessment does not necessarily mean women do, or do not, display symptoms of pelvic floor disorders or pain. It is presently unknown whether knowledge of whether a PV muscle tear is likely to be present could help contribute to preventing the development of pelvic floor disorders later in life, or whether existing strategies for treating pelvic floor disorders are optimal or even appropriate in the presence of a PV muscle tear.

An association between PV muscle tear and both underactive pelvic muscle contraction ${ }^{24}$ and vaginal closure force reduction has already been reported., ${ }^{9,23,25}$ With PV muscle tear, the PV muscle logically loses its functional effectiveness due to the avulsion from its origin. It follows that pelvic muscle exercises, consisting of repetitive contractions with the goal to strengthen the $\mathrm{PV}$ muscle, might not be effective if the muscle is torn away from its origin and is chronic. ${ }^{9}$ Therefore, it appears worthwhile to assess the PV muscle for tear status before prescribing pelvic muscle exercises as a treatment. This is similar to evaluating for anterior cruciate ligament tear status, via the simple anterior drawer test, before embarking on a selected program of physical therapy for knee disorders. Need for follow-up MRI for diagnostic certainty would be dependent on whether care choices are dependent on the precision of diagnosis. Confirmatory diagnosis provides the basis for physical therapy decisions, including the rationale for modifications to physical activity. Evaluation of "strength" (vaginal closure force), without attention to a potential tear, may fail to reveal the reasons for a loss of vaginal closure force. Women with torn PV muscle might best be instructed, for instance, in lifestyle factors and even pessary support for the pelvic organs during high-impact activities.

There are several limitations to this study. These participants were selected based on risk factors for PV muscle tear rather than presence of symptoms of urinary incontinence or pelvic organ prolapse. Hence, the value of PV muscle body integrity palpatory assessment for symptomatic women could not be explored in the context of this study design. Neither interrater nor intrarater reliability could be evaluated in this study, due to cost of a second evaluator and in consideration that the parent study's two timepoints were separated by 8 months of post-birth vaginal changes. ${ }^{9}$ The odds ratio was not adjusted for other confounding obstetric risk factors for PV muscle tear. Lastly, the sample size was relatively small, and larger studies are needed to confirm the findings and determine inter and intrareliability. 


\section{5 | CONCLUSIONS}

Index finger palpatory assessment at the site of the PV muscle body can be used to estimate the odds of PV muscle tear in postpartum women with known PV muscle tear risk factors. Using index finger palpatory assessment clinically is a starting point for assessment and decision-making about appropriateness of otherwise routinely prescribed pelvic muscle strengthening programs, which currently are typically recommended without regard to consideration of PV muscle tear status.

\section{ACKNOWLEDGEMENTS}

The authors would like to thank Dr. John DeLancey, who offered use in this article of the image he created showing a diagrammatic portrayal of index finger placement for palpatory assessment of the pubovisceral muscle body. Heather van Doren, senior medical editor at Arbor Research Collaborative for Health, provided editorial assistance on this manuscript. The Evaluating Maternal Recovery from Labor and Delivery (EMRLD) study is grant-supported by the National Institutes of Health (NIH) through the Office for Research on Women's Health Specialized Center of Research (SCOR) on Sex and Gender Factors Affecting Women's Health and National Institute on Child and Human Development (grant \#P50 HD044406 002) and the National Institute on Child and Human Development (grant \#R21 HD049818). The content of this manuscript is solely the responsibility of the authors and does not necessarily represent the official views of the NIH or its institutes. The sponsor had no involvement in study design; collection, analysis, and interpretation of data; writing of the report; or decision to submit the article for publication.

\section{CONFLICT OF INTERESTS}

The authors declare that there are no conflict of interests.

\section{REFERENCES}

1. DeLancey JOL, Kearney R, Chou Q, et al. The appearance of levator ani muscle abnormalities in magnetic resonance images after vaginal delivery. Obstet Gynecol. 2003;101:46-53.

2. Dietz HP, Steensma AB. The prevalence of major abnormalities of the levator ani in urogynaecological patients. BJOG. 2006;113:225-230.

3. Kim J, Betschart C, Ramanah R, et al. Anatomy of the pubovisceral muscle origin: macroscopic and microscopic findings within the injury zone. Neurourol Urodyn. 2015;34:774-780.

4. Lien KC, Mooney B, DeLancey JOL, et al. Levator ani muscle stretch induced by simulated vaginal birth. Obstet Gynecol. 2004;103:31-40
5. Tracy PV, DeLancey JOL, Ashton-Miller JA. A geometric capacity-demand analysis of maternal levator muscle stretch required for vaginal delivery. J Biomech Eng. 2016;138(2): 0210011-02100112.

6. Dietz HP, Lanzarone V. Levator trauma after vaginal delivery. Obstet Gynecol. 2005;106:707-712. 02100112

7. Shek K, Dietz HP. Intrapartum risk factors for levator trauma. BJOG. 2010;117:1485-1492.

8. Valsky DV, Lipschuetz M, Bord A, et al. Fetal head circumference and length of second stage of labor are risk factors for levator ani muscle injury, diagnosed by 3-dimensional transperineal ultrasound in primiparous women. Am J Obstet Gynecol. 2009;201:e1-e7.

9. Miller JM, Low LK, Zielinski R, et al. Evaluating maternal recovery from labor and delivery: bone and levator ani injuries. Am J Obstet Gynecol. 2015;213:e1-e11.

10. Kearney R, Fitzpatrick M, Brennan S, et al. Levator ani injury in primiparous women with forceps delivery for fetal distress, forceps for second stage arrest, and spontaneous delivery. Int $J$ Gynecol Obstet. 2010;111:19-22.

11. Kearney R, Miller JM, Ashton-Miller JA, et al. Obstetric factors associated with levator ani muscle injury after vaginal birth. Obstet Gynecol. 2006;107:144-149.

12. Low KL, Zielinski R, Tao Y, et al. Predicting birth-related levator ani tear severity in primiparous women: Evaluating maternal recovery from labor and delivery (EMRLD study). Open J Obstet Gynecol. 2014;4:266-278.

13. Brandon C, Jacobson J, Low LK, et al. Pubic bone injuries in primiparous women: magnetic resonance imaging in detection and differential diagnosis of structural injury. Ultrasound in Obstet Gynecol. 2012;39:444-451.

14. Schwertner-Tiepelmann N, Thakar R, Sultan AH, et al. Obstetric levator ani muscle injuries: current status. Ultrasound in Obstet Gynecol. 2012;39:372-383.

15. van Delft K, Schwertber-Tiepelmann N, Thakar R, et al. Interrater reliability of assessment of levator ani muscle strength and attachment to the pubic bone in nulliparous women. Ultrasound in Obstet Gynecol. 2013;42:341-346.

16. Brink CA, Sampselle CM, Wells TJ, et al. A digital test for pelvic muscle strength in older women with urinary incontinence. Nurs Res. 1989;38:196-199.

17. Agresti A. Analysis of ordinal categorical data. 2nd ed. Hoboken, NJ: John Wiley \& Sons; 2010.

18. van Delft K, Sultan AH, Thakar R, et al. Agreement between palpation and transperineal and endovaginal ultrasound in the diagnosis of levator ani avulsion. Int Urogynecol $J$. 2015;26:33-39.

19. Yan Y, Dou C, Wang X, et al. Combination of tomographic ultrasound imaging and three-dimensional magnetic resource imaging-based model to diagnose postpartum levator avulsion. Sci Rep. 2017;7:1-9.

20. Gainey HL. Postpartum observation of pelvic tissue damage. Am J Obstet Gynecol. 1943;45:457-466.

21. Gainey HL. Postpartum observation of pelvic tissue damage: further studies. Am J Obstet Gynecol. 1955;70:800-807.

22. Lammers K, Futterer JJ, Prokop M, et al. Diagnosing pubovisceral avulsion: a systematic review of the clinical relevance of a prevalent anatomical defect. Int Urogynecol J. 2012;23:1653-1664. 
23. Dietz HP, Shek C. Validity and reproducibility of the digital detection of levator trauma. Int Urogynecol J. 2008;19:1097-1101.

24. Steensma AB, Konstantinovic ML, Burger CW, et al. Prevalence of major levator abnormalities in symptomatic patients with an underactive pelvic floor contraction. Int Urogynecol J. 2010; 21:861-867.

25. DeLancey JOL, Morgan DM, Fenner DE, et al. Comparison of levator ani muscle defects and function in women with and without pelvic organ prolapse. Obstet Gynecol. 2007;109:295-302.
How to cite this article: Sheng Y, Low LK, Liu X, Ashton-Miller JA, Miller JM. Association of index finger palpatory assessment of pubovisceral muscle body integrity with MRI-documented tear.

Neurourology and Urodynamics. 2019;38:1120-1128. https://doi.org/10.1002/nau.23967 